\title{
Fast parallel factor decomposition technique for coherently distributed source localization
}

\author{
CHENG Qianlin, ZHANG Xiaofei", and CAO Renzheng \\ College of Electronic and Information Engineering, Nanjing University of Aeronautics and Astronautics, Nanjing 210016, China
}

\begin{abstract}
This paper links parallel factor (PARAFAC) analysis to the problem of nominal direction-of-arrival (DOA) estimation for coherently distributed (CD) sources and proposes a fast PARAFACbased algorithm by establishing the trilinear PARAFAC model. Relying on the uniqueness of the low-rank three-way array decomposition and the trilinear alternating least squares regression, the proposed algorithm achieves nominal DOA estimation and outperforms the conventional estimation of signal parameter via rotational technique CD (ESPRIT-CD) and propagator method CD (PM-CD) methods in terms of estimation accuracy. Furthermore, by means of the initialization via the propagator method, this paper accelerates the convergence procedure of the proposed algorithm with no estimation performance degradation. In addition, the proposed algorithm can be directly applied to the multiple-source scenario, where sources have different angular distribution shapes. Numerical simulation results corroborate the effectiveness and superiority of the proposed fast PARAFAC-based algorithm.
\end{abstract}

Keywords: source localization, coherently distributed (CD) source, parallel factor analysis, propagator method (PM), trilinear decomposition.

DOI: $10.21629 /$ JSEE.2018.04.01

\section{Introduction}

In general, most of traditional direction-of-arrival (DOA) estimation methods are developed for far-field point sources which correspond to the one-ray propagation. However, in actual implementation, due to multipath phenomena, the signal will impinge on the array through many rays which are reflected or scattered from the vicinity of the sources. Consequently, a distributed source model would be more appropriate [1-4], in which the angular spread is also taken into consideration. For decades,

Manuscript received March 24, 2017.

*Corresponding author.

This work was supported by the National Natural Science Foundation of China (61371169; 61601167), the Jiangsu Natural Science Foundation (BK20161489), the open research fund of State Key Laboratory of Millimeter Waves, Southeast University (K201826), and the Fundamental Research Funds for the Central Universities (NE2017103). the localization of the distributed sources [5] has attracted much attention in the field of array signal processing. Distributed source localization techniques have been applied in wireless communication [6], radar [7], sonar [8] and other fields. According to the correlation among different rays [5], the distributed source can be classified into two different types: incoherently distributed (ID) source or coherently distributed (CD) source. For the ID source case we consider, the signal components from different rays are supposed to be completely uncorrelated [5], whereas for the CD source case, these components are completely correlated [9].

Directly applying the DOA estimation methods for the point sources, e.g., multiple signal classification (MUSIC) [10], estimation of signal parameter via rotational technique (ESPRIT) [11] and propagator method (PM) into the CD sources scenario will induce significant degradation in the estimation accuracy [12]. Several works have been proposed by extending the conventional methods for the point sources case, e.g., the distributed signal parameter estimator (DSPE) [5] and ESPRIT-CD [13] are variants of MUSIC and ESPRIT, respectively. Several spectral peak searching algorithms for distributed sources have been developed in $[2-4,14,15]$. To relieve the heavy computational load due to multi-dimensional search in [2$5,14,15]$, many extensions using the subspace-based estimator have been proposed in [16-23]. However, the search-free technique in [23] can only be applied in the case of the single source. In addition, some other methods such as the generalized capon method and the maximum likelihood method have also been proposed in [24,25].

Parallel factor (PARAFAC) analysis is a typical method for low-rank decomposition of three-way and higher-way arrays. In array signal processing and communications [26,27], it has been widely adopted and can be treated as a generalization of ESPRIT [11] or joint approximate diagonalization [28]. Unlike the matrix, i.e., the two-way array bilinear model, which is exploited in most DOA estimators, the PARAFAC model imposes an attractive fea- 
ture: its inherent uniqueness, which means the PARAFAC model suffers from no rotational freedom even under mild conditions. Moreover, this uniqueness allows a much more straightforward solution for parameter estimation in signal processing [26,27]. However, previous related works are confined to the point source case.

In this paper, we link the problem of nominal DOA estimation of CD sources to PARAFAC analysis, and propose a fast PARAFAC analysis-based algorithm consequently. We accelerate the convergence procedure of the proposed algorithm by initializing the estimation parameters via PM. Specifically, as compared with the conventional PARAFAC, the proposed algorithm can achieve a fast convergence with no estimation performance degradation, which endows it a much lower complexity. Meanwhile, the proposed algorithm outperforms the conventional ESPRIT-CD [13] and PM-CD [22] algorithm in terms of estimation accuracy. In addition, simulation results are provided to demonstrate the effectiveness and superiority of the proposed algorithm.

Hereby the main contributions of this paper are presented as follows:

(i) The problem of nominal DOA estimation for CD sources is linked to PARAFAC decomposition technique, which has not been studied in the previous literatures, as far as we know.

(ii) We exploit PM to initialize the estimation parameter to speed up the convergence process of the proposed algorithm, which remarkably reduces the computational complexity.

(iii) We conduct the proposed fast PARAFAC algorithm in data domain to resolve the nominal DOA estimation for CD sources while the subspace-based methods involve the inefficient eigenvalue decomposition.

The remainder of this paper is organized as follows. Section 2 introduces the data model. We propose the fast PARAFAC algorithm in Section 3. In Section 4, complexity analysis and advantages of the fast PARAFAC algorithm are given. Numerical simulations are provided in Section 5 and we conclude this paper in Section 6.

Notations: We use lower-case (upper-case) bold characters to denote vectors (matrix). $(\cdot)^{\mathrm{T}},(\cdot)^{\mathrm{H}},(\cdot)^{-1},(\cdot)^{+}$denote the transpose, the conjugate transpose, inverse and pseudo-inverse of a matrix or vector, respectively. $\operatorname{diag}(\cdot)$ denotes a diagonal matrix that uses the elements of the matrix as its diagonal elements. $\mathrm{E}\{\cdot\}$ is the statistical expectation operator. $\odot$ denotes the Khatri-Rao product. $D_{m}(\cdot)$ denotes a diagonal matrix consisting of the $m$ th row of the matrix. angle $(\cdot)$ denotes phase operator.

\section{Data model}

The array geometry we consider herein is a multiple- invariance array as shown in Fig. 1. It is composed of $P$ identical uniform linear arrays (ULAs) with a constant displacement between two adjacent subarrays, where each ULA consists of $M$ elements with the same inter-element spacing. For the ease of illustration, we assume that all the subarrays are linear and are placed along $x$-axis.



Fig. 1 Array structure

Set the first sensor of subarray 1 as the reference point and suppose that the displacement between two adjacent subarrays is $d$. If we denote the coordinates of the first subarray as $\left\{x_{1, m}\right\}_{m=1}^{M}$, then the coordinate of the $m$ th sensor in the $p$ th subarray is $x_{p, m}=x_{1, m}+(p-1) d(m=$ $1, \ldots, M ; p=1, \ldots, P)$. Assume that a wave field of $K$ narrow-band far-field $C D$ sources impinges on the array and $K \leqslant M$. The output of the first subarray [5] can be expressed as

$$
\tilde{\boldsymbol{r}}_{1}(t)=\sum_{k=1}^{K} \int_{-\pi / 2}^{\pi / 2} \boldsymbol{a}(\theta) s_{k}\left(\theta, t ; \boldsymbol{\eta}_{k}\right) \mathrm{d} \theta+\boldsymbol{n}_{1}(t)
$$

where $\boldsymbol{a}(\theta)=\left[\mathrm{e}^{\mathrm{j} 2 \pi x_{1,1} / \lambda \sin \theta}, \ldots, \mathrm{e}^{\mathrm{j} 2 \pi x_{1, M} / \lambda \sin \theta}\right]^{\mathrm{T}} \mathrm{de}-$ notes the direction vector of angle $\theta ; s_{k}\left(\theta, t ; \boldsymbol{\eta}_{k}\right)$ is the angular density; $\boldsymbol{\eta}_{k}=\left[\begin{array}{ll}\theta_{k} & \sigma_{k}\end{array}\right]$ represents the location parameter vector with the nominal DOA $\theta_{k}$ and the angular spread $\sigma_{k} ; \boldsymbol{n}_{1}(t)$ denotes the additive zero-mean white Gaussian noise vector with variance $\sigma_{n}^{2}$. Note that the in$\operatorname{dex} k$ in $s_{k}\left(\theta, t ; \boldsymbol{\eta}_{k}\right)$ and $\boldsymbol{\eta}_{k}=\left[\begin{array}{ll}\theta_{k} & \sigma_{k}\end{array}\right]$ denotes the $k$ th source; $t$ denotes the time index.

The angular signal density $s_{k}\left(\theta, t ; \boldsymbol{\eta}_{k}\right)[13]$ can be represented by

$$
s_{k}\left(\theta, t ; \boldsymbol{\eta}_{k}\right)=v_{k}(t) g_{k}\left(\theta ; \boldsymbol{\eta}_{k}\right)
$$

where $v_{k}(t)$ stands for the complex random signal of the $k$ th source, and $g_{k}\left(\theta ; \boldsymbol{\eta}_{k}\right)$ is a deterministic symmetric angular distribution function. Note that the index $k$ in $g_{k}\left(\theta ; \boldsymbol{\eta}_{k}\right)$ implies that different sources may have different angular distributions. 
Then (1) can be rewritten as

$$
\tilde{\boldsymbol{r}}_{1}(t)=\boldsymbol{B}_{1}(\boldsymbol{\eta}) \boldsymbol{v}(t)+\boldsymbol{n}_{1}(t)
$$

where

$$
\begin{gathered}
\boldsymbol{B}_{1}(\boldsymbol{\eta})=\left[\boldsymbol{b}\left(\boldsymbol{\eta}_{1}\right), \boldsymbol{b}\left(\boldsymbol{\eta}_{2}\right), \ldots, \boldsymbol{b}\left(\boldsymbol{\eta}_{K}\right)\right] \\
\boldsymbol{v}(t)=\left[v_{1}(t), v_{2}(t), \ldots, v_{K}(t)\right]^{\mathrm{T}} \\
\boldsymbol{\eta}=\left[\boldsymbol{\eta}_{1}, \boldsymbol{\eta}_{2}, \ldots, \boldsymbol{\eta}_{K}\right]
\end{gathered}
$$

and

$$
\boldsymbol{b}\left(\boldsymbol{\eta}_{k}\right)=\int_{-\pi / 2}^{\pi / 2} \boldsymbol{a}(\theta) g_{k}\left(\theta ; \boldsymbol{\eta}_{k}\right) \mathrm{d} \theta .
$$

Define matrix $\boldsymbol{G} \in \mathbf{C}^{P \times K}$ as

$$
\boldsymbol{G}^{\mathrm{T}}=\left[\boldsymbol{G}_{1}, \ldots, \boldsymbol{G}_{P}\right]
$$

where $\boldsymbol{G}_{p}=\left[\mathrm{e}^{\mathrm{j} 2 \pi d(p-1) \sin \theta_{1} / \lambda}, \ldots, \mathrm{e}^{\mathrm{j} 2 \pi d(p-1) \sin \theta_{K} / \lambda}\right]^{\mathrm{T}}$.

As proved in [13], when $P d \leqslant \lambda$ and the angular distribution is symmetric, an approximate shift-invariant property [13] holds among the array manifold matrix of all subarrays, i.e.,

$$
\boldsymbol{B}_{p}(\boldsymbol{\eta})=\boldsymbol{B}_{1}(\boldsymbol{\eta}) \boldsymbol{D}_{p}(\boldsymbol{G})
$$

where $\boldsymbol{B}_{p}$ denotes the direction matrix of the $p$ th subarray and $\boldsymbol{D}_{p}(\cdot)$ stands for a diagonal matrix whose elements on the main diagonal are the $p$ th row of the bracketed matrix.

Hence, when $P d \leqslant \lambda$, the received data of the entire array can be presented in the form of matrix as

$$
\begin{aligned}
\widetilde{\boldsymbol{r}}=\left[\begin{array}{c}
\widetilde{\boldsymbol{r}}_{1} \\
\widetilde{\boldsymbol{r}}_{2} \\
\vdots \\
\widetilde{\boldsymbol{r}}_{P}
\end{array}\right]= & {\left[\begin{array}{c}
\boldsymbol{B}_{1} D_{1}(\boldsymbol{G}) \\
\boldsymbol{B}_{1} D_{2}(\boldsymbol{G}) \\
\vdots \\
\boldsymbol{B}_{1} D_{P}(\boldsymbol{G})
\end{array}\right] \boldsymbol{v}+\left[\begin{array}{c}
\boldsymbol{n}_{1} \\
\boldsymbol{n}_{2} \\
\vdots \\
\boldsymbol{n}_{P}
\end{array}\right]=} \\
& {\left[\boldsymbol{G} \odot \boldsymbol{B}_{1}\right] \boldsymbol{v}+\boldsymbol{n} }
\end{aligned}
$$

where $\tilde{\boldsymbol{r}}_{p}(p=1, \ldots, P)$ denotes the output of the $p$ th subarray, $\boldsymbol{n}=\left[\boldsymbol{n}_{1}^{\mathrm{T}}, \ldots, \boldsymbol{n}_{P}^{\mathrm{T}}\right]^{\mathrm{T}}$ denotes the noise vector, and the notations $\boldsymbol{\eta}$ and $t$ are omitted for simplicity.

Collect $Q$ snapshots and formulate an $M P \times Q$ matrix as

$$
\widetilde{\boldsymbol{R}}=\left[\widetilde{\boldsymbol{R}}_{1}^{\mathrm{T}}, \widetilde{\boldsymbol{R}}_{2}^{\mathrm{T}}, \ldots, \widetilde{\boldsymbol{R}}_{P}^{\mathrm{T}}\right]^{\mathrm{T}}=\left[\boldsymbol{G} \odot \boldsymbol{B}_{1}\right] \boldsymbol{V}+\boldsymbol{N}
$$

where $\boldsymbol{V}=\left[\boldsymbol{v}\left(t_{1}\right), \ldots, \boldsymbol{v}\left(t_{Q}\right)\right] \in \mathbf{C}^{K \times Q}$ is the $K \times Q$ source signal matrix, and $N \in \mathbf{C}^{M \times Q}$ is the $M \times Q$ noise matrix. Define the noise-free part of $\widetilde{\boldsymbol{R}}$ as

$$
\boldsymbol{R}=\left[\boldsymbol{R}_{1}^{\mathrm{T}}, \boldsymbol{R}_{2}^{\mathrm{T}}, \ldots, \boldsymbol{R}_{P}^{\mathrm{T}}\right]^{\mathrm{T}}=\left[\boldsymbol{G} \odot \boldsymbol{B}_{1}\right] \boldsymbol{V}
$$

where

$$
\boldsymbol{R}_{p}=\boldsymbol{B}_{1} D_{p}(\boldsymbol{G}) \boldsymbol{V}, \boldsymbol{R}_{p} \in \mathbf{C}^{M \times Q}, \quad p=1,2, \ldots P .
$$

\section{Proposed algorithm}

\subsection{PARAFAC modeling}

The $(m, q)$ th element of $\boldsymbol{R}_{p}$, denoted by $r_{m, q, p}$ [26] can be written as a sum of triple products

$$
r_{m, q, p}=\sum_{k=1}^{K}\left[\boldsymbol{B}_{1}\right]_{m, k}[\boldsymbol{G}]_{p, k}[\boldsymbol{V}]_{k, q}
$$

where $\left[\boldsymbol{B}_{1}\right]_{m, k}$ stands for the $(m, k)$ th entry of $\boldsymbol{B}_{1}$, and similarly for the others. Equation (14) is also known as the PARAFAC model or trilinear decomposition of $r_{m, q, p}$ [26]. Consequently, we define a three-way matrix $\boldsymbol{R}$ with element $r_{m, q, p}$.

As shown in Fig. 2, the noise-free observation signal is arranged in a three-way diversity space, i.e., spatial way, temporal way and invariance way [26]. Equation (13) can be regarded as a slice of the three-way matrix $\boldsymbol{R}$ versus $P$ slabs along the invariance dimension. Due to the symmetry property of the PARAFAC model in (14), two different matrix rearrangements $\boldsymbol{Y}$ and $\boldsymbol{Z}$ can be constructed, which can be interpreted as slicing the three-way matrix $\boldsymbol{R}$ along the other two dimensions. To be more specific,

$$
\begin{gathered}
\boldsymbol{Y}=\left[\boldsymbol{Y}_{1}^{\mathrm{T}}, \boldsymbol{Y}_{2}^{\mathrm{T}}, \ldots, \boldsymbol{Y}_{M}^{\mathrm{T}}\right]^{\mathrm{T}}=\left[\boldsymbol{B}_{1} \odot \boldsymbol{V}^{\mathrm{T}}\right] \boldsymbol{G}^{\mathrm{T}} \\
\boldsymbol{Z}=\left[\boldsymbol{Z}_{1}^{\mathrm{T}}, \boldsymbol{Z}_{2}^{\mathrm{T}}, \ldots, \boldsymbol{Z}_{Q}^{\mathrm{T}}\right]^{\mathrm{T}}=\left[\boldsymbol{V}^{\mathrm{T}} \odot \boldsymbol{G}\right] \boldsymbol{B}_{1}^{\mathrm{T}}
\end{gathered}
$$

where

$$
\begin{gathered}
\boldsymbol{Y}_{m}=\boldsymbol{V}^{\mathrm{T}} D_{M}\left(\boldsymbol{B}_{1}\right) \boldsymbol{G}^{\mathrm{T}}, \quad m=1,2, \ldots, M \\
\boldsymbol{Z}_{q}=\boldsymbol{G} D_{q}\left(\boldsymbol{V}^{\mathrm{T}}\right) \boldsymbol{B}_{1}^{\mathrm{T}}, \quad q=1,2, \ldots, Q .
\end{gathered}
$$

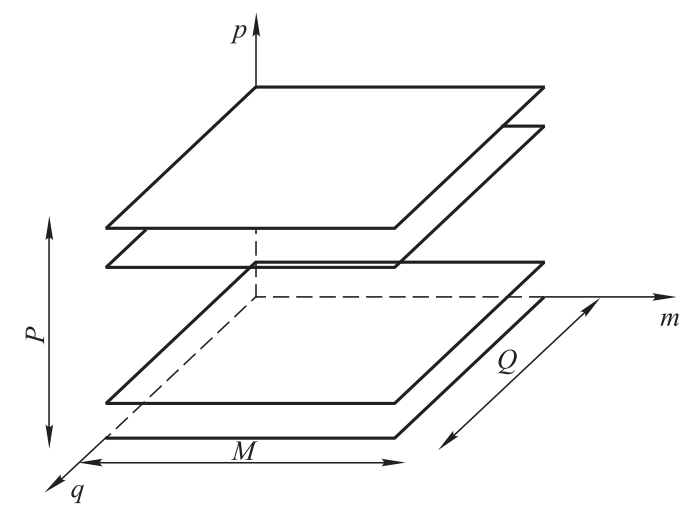

Fig. 2 PARAFAC model

The conventional PARAFAC algorithm uses the random initialization, which leads to a slow convergence and a heavy computational complexity. In order to tackle the problems, we utilize the PM method to conduct the initialization, which can speed up the convergence and hence reduce the computational complexity. And the specific procedure is particularly illustrated in Section 3.2. 


\subsection{Initialization with PM method}

In this section, we firstly exploit PM to get the rough estimation of nominal DOAs and then construct the initializations, $\boldsymbol{B}_{1}$ and $\boldsymbol{G}$.

Assume that the first $K$ rows of $\boldsymbol{A}=\boldsymbol{G} \odot \boldsymbol{B}_{1}$ are linearly independent. Firstly, we can define and partition $\boldsymbol{A}$ as

$$
\boldsymbol{A}=\left[\begin{array}{l}
\boldsymbol{A}_{1} \\
\boldsymbol{A}_{2}
\end{array}\right]
$$

where $\boldsymbol{A}_{1}$, which is assumed to be a nonsingular matrix, denotes the first $K$ rows of $\boldsymbol{A}$ while $\boldsymbol{A}_{2}$ denotes the remaining $M P-K$ rows of $\boldsymbol{A}$.

There exists a linear operator between $\boldsymbol{A}_{1}$ and $\boldsymbol{A}_{2}$, which can be expressed as

$$
\boldsymbol{A}_{2}=\boldsymbol{P}_{c} \boldsymbol{A}_{1}
$$

The propagator $\boldsymbol{P}_{c}$ [29] can be computed by

$$
\boldsymbol{P}_{c}=\left(\widetilde{\boldsymbol{R}}_{1} \widetilde{\boldsymbol{R}}_{1}^{\mathrm{H}}\right)^{-1} \widetilde{\boldsymbol{R}}_{1} \widetilde{\boldsymbol{R}}_{2}^{\mathrm{H}}
$$

where $\widetilde{\boldsymbol{R}}_{1}$ and $\widetilde{\boldsymbol{R}}_{2}$ denote the first $K$ rows and the remaining rows of the received signal $\widetilde{\boldsymbol{R}}$ in (11), respectively.

Define an $M P \times K$ matrix [34]

$$
\boldsymbol{P}=\left[\begin{array}{c}
\boldsymbol{I}_{K} \\
\boldsymbol{P}_{c}
\end{array}\right]
$$

where $\boldsymbol{I}_{K} \in \mathbf{R}^{K \times K}$ is an identity matrix. According to (19) and (20), it is easy to obtain [30] that

$$
\begin{gathered}
\boldsymbol{P} \boldsymbol{A}_{1}=\left[\begin{array}{c}
\boldsymbol{I}_{K} \\
\boldsymbol{P}_{c}
\end{array}\right] \boldsymbol{A}_{1}=\boldsymbol{A} . \\
{\left[\begin{array}{c}
\boldsymbol{P}_{a} \\
\boldsymbol{P}_{b}
\end{array}\right] \boldsymbol{A}_{1}=\left[\begin{array}{c}
\boldsymbol{A}_{a} \\
\boldsymbol{A}_{b}
\end{array}\right]=\left[\begin{array}{c}
\boldsymbol{A}_{a} \\
\boldsymbol{A}_{a} \boldsymbol{\Phi}_{r}
\end{array}\right]}
\end{gathered}
$$

where $\boldsymbol{A}_{a}$ and $\boldsymbol{A}_{b}$ denote the first $M P-P$ rows and the last $M P-P$ rows of $\boldsymbol{A}$, respectively. $\boldsymbol{P}_{a}$ stands for the first $M P-P$ rows of $\boldsymbol{P}$ and $\boldsymbol{P}_{b}$ stands for the last $M P-P$ rows of $\boldsymbol{P}$.

Then we can achieve the following relationship:

$$
\boldsymbol{P}_{a}^{+} \boldsymbol{P}_{b}=\boldsymbol{A}_{1} \boldsymbol{\Phi}_{r} \boldsymbol{A}_{1}^{-1}
$$

Thus the initial rough estimation of nominal DOAs can be obtained by performing eigenvalue decomposition on $\boldsymbol{P}_{a}^{+} \boldsymbol{P}_{b}$. Then the initializations $\boldsymbol{G}$ and $\boldsymbol{B}_{1}$ can be constructed.

\subsection{Trilinear decomposition}

Given the noisy three-way observation data $\widetilde{\boldsymbol{R}}$, we adopt the trilinear alternating least squares (TALS) method [26] to perform trilinear decomposition and estimate $\boldsymbol{B}_{1}, \boldsymbol{G}$ and
$\boldsymbol{V}$. Then we discuss the sufficient and necessary condition for unique estimation compactly (see [31] for detail).

Theorem 1 [31] Consider the set of $P$ matrices $\boldsymbol{R}_{p}=$ $\boldsymbol{B}_{1} D_{p}(\boldsymbol{G}) \boldsymbol{V}, p=1,2, \ldots, P, \boldsymbol{B}_{1} \in \mathbf{C}^{M \times K}, \boldsymbol{G} \in$ $\mathbf{C}^{P \times K}$, and $\boldsymbol{V} \in \mathbf{C}^{K \times Q}$. When $\boldsymbol{B}_{1}, \boldsymbol{G}, \boldsymbol{V}$ are full $k$-rank, which is generically satisfied in practice, if the parameter identifiability satisfies

$$
\min (M, K)+\min (P, K)+\min (Q, K) \geqslant 2 K+2,
$$

then $\boldsymbol{B}_{1}, \boldsymbol{G}, \boldsymbol{V}$ can be uniquely identified from $\boldsymbol{R}_{p}$ (up to permutation and scale ambiguity).

Given initial estimates, TALS proceeds to update $\boldsymbol{B}_{1}, \boldsymbol{G}$ and $\boldsymbol{V}$ using the least square conditioned on previously obtained estimates of the remaining parameters in a roundrobin fashion. The update is repeated until convergence, which is globally monotoned and guaranteed [26].

According to (11), the least squares fitting amounts to

$$
\min _{\boldsymbol{B}_{1}, \boldsymbol{G}, \boldsymbol{V}}\left\|\widetilde{\boldsymbol{R}}-\left[\boldsymbol{G} \odot \boldsymbol{B}_{1}\right] \boldsymbol{V}\right\|_{F}^{2}
$$

where $\|\cdot\|_{F}$ stands for the Frobenius norm. It follows the conditional least squares update for $\boldsymbol{V}$, which can be presented by

$$
\widehat{\boldsymbol{V}}=\left[\widehat{\boldsymbol{G}} \odot \widehat{\boldsymbol{B}}_{1}\right]^{+} \widetilde{\boldsymbol{R}}
$$

where $\widehat{\boldsymbol{B}}_{1}$ and $\widehat{\boldsymbol{G}}$ denote the previous estimates of $\boldsymbol{B}_{1}$ and $\boldsymbol{G}$, respectively. Similarly, the least square fitting of the second slicing (15) is equivalent to

$$
\min _{\boldsymbol{B}_{1}, \boldsymbol{G}, \boldsymbol{V}}\left\|\tilde{\boldsymbol{Y}}-\left[\boldsymbol{B}_{1} \odot \boldsymbol{V}\right] \boldsymbol{G}^{\mathrm{T}}\right\|_{F}^{2}
$$

where $\tilde{\boldsymbol{Y}}=\left[\tilde{\boldsymbol{Y}}_{1}^{\mathrm{T}}, \tilde{\boldsymbol{Y}}_{2}^{\mathrm{T}}, \ldots \tilde{\boldsymbol{Y}}_{M}^{\mathrm{T}}\right]^{\mathrm{T}}$ and $\tilde{\boldsymbol{Y}}_{m}(m=1, \ldots, M)$ are the noisy slabs along the spatial dimension. Subsequently, the least square update for $\boldsymbol{G}$ is

$$
\widehat{\boldsymbol{G}}^{\mathrm{T}}=\left[\widehat{\boldsymbol{B}}_{1} \odot \widehat{\boldsymbol{V}}^{\mathrm{T}}\right]^{+} \widetilde{\boldsymbol{Y}} .
$$

Finally, from the third way of slicing the three-way data (16), the least square fitting is given by

$$
\min _{\boldsymbol{B}_{1}, \boldsymbol{G}, \boldsymbol{V}}\left\|\widetilde{\boldsymbol{Z}}-\left[\widehat{\boldsymbol{V}}^{\mathrm{T}} \odot \widehat{\boldsymbol{G}}\right] \widehat{\boldsymbol{B}}_{1}\right\|_{F}^{2}
$$

where $\widetilde{\boldsymbol{Z}}=\left[\widetilde{\boldsymbol{Z}}_{1}^{\mathrm{T}}, \widetilde{\boldsymbol{Z}}_{2}^{\mathrm{T}}, \ldots, \widetilde{\boldsymbol{Z}}_{Q}^{\mathrm{T}}\right]^{\mathrm{T}}$ with $\widetilde{\boldsymbol{Z}}_{q}(q=$ $1,2, \ldots, Q)$ is the noisy slabs along the temporal dimension. Consequently, the least square update for $\boldsymbol{B}_{1}$ is found as

$$
\widehat{\boldsymbol{B}}_{1}^{\mathrm{T}}=\left[\widehat{\boldsymbol{V}}^{\mathrm{T}} \odot \widehat{\boldsymbol{G}}\right]^{+} \widetilde{\boldsymbol{Z}} .
$$

Due to the initialization implemented by PM, the proposed algorithm can achieve a faster convergence compared with the conventional PARAFAC.

\subsection{Nominal DOA estimation}

By means of TALS, we can obtain the estimates of $\boldsymbol{B}_{1}, \boldsymbol{G}$ and $\boldsymbol{V}$, which are given by

$$
\widehat{B}_{1}=B_{1} \Pi \Omega_{1}+E_{1}
$$




$$
\begin{aligned}
& \widehat{\boldsymbol{G}}=\boldsymbol{G} \boldsymbol{\Pi} \boldsymbol{\Omega}_{2}+\boldsymbol{E}_{2} \\
& \widehat{\boldsymbol{V}}=\boldsymbol{V} \boldsymbol{\Pi} \boldsymbol{\Omega}_{3}+\boldsymbol{E}_{3}
\end{aligned}
$$

where $\boldsymbol{\Pi}$ is a permutation matrix, $\boldsymbol{E}_{1}, \boldsymbol{E}_{2}, \boldsymbol{E}_{3}$ are corresponding error matrices, and $\boldsymbol{\Omega}_{1}, \boldsymbol{\Omega}_{2}, \boldsymbol{\Omega}_{3}$ are diagonal scaling matrices with $\Omega_{1} \Omega_{2} \Omega_{3}=I$. In trilinear decomposition, the permutation and scale ambiguity are inherent, which have no difference for our source localization, wherein the scale ambiguity can be eliminated by normalization.

Let $\boldsymbol{h}(k)$ denote the phase angles of the $k$ th column of $\boldsymbol{G}$, which is given by

$$
\boldsymbol{h}(k)=\left[\begin{array}{c}
0 \\
2 \pi \mathrm{d} \sin \theta_{k} / \lambda \\
\vdots \\
2 \pi(P-1) \mathrm{d} \sin \theta_{k} / \lambda
\end{array}\right]^{\mathrm{T}} .
$$

After obtaining the estimated $\widehat{G}$, we can normalize the columns of $\widehat{G}$ and take the phase angle which is denoted by $\widehat{\boldsymbol{H}}$. Subsequently, we employ the least square principle to estimate nominal DOAs. Define the least square fitting as

$$
\boldsymbol{U} \boldsymbol{w}=\widehat{\boldsymbol{H}}
$$

where $\boldsymbol{w} \in \mathbf{C}^{2 \times K}$ and

$$
\boldsymbol{U}=\left[\begin{array}{cc}
1 & 0 \\
1 & 2 \pi d / \lambda \\
\vdots & \vdots \\
1 & 2 \pi(P-1) d / \lambda
\end{array}\right]
$$

The least square solution for $\boldsymbol{w}$ is

$$
\boldsymbol{w}=\left(\boldsymbol{U}^{\mathrm{T}} \boldsymbol{U}\right)^{-1} \boldsymbol{U}^{\mathrm{T}} \widehat{\boldsymbol{H}} .
$$

Hence the $K$ estimates of nominal DOAs, namely $\left\{\widehat{\theta}_{k}\right\}_{k=1}^{K}$ can be calculated by

$$
\widehat{\theta}_{k}=\arcsin \left(\boldsymbol{w}_{2,:}\right), \quad k=1,2, \ldots, K
$$

where $\boldsymbol{w}_{2, \text { : }}$ denotes the second row of $\boldsymbol{w}$.

\subsection{Detailed steps of the fast PARAFAC algorithm}

The detailed steps of the fast PARAFAC algorithm are presented as follows:

Step 1 Obtain the estimated propagator $\boldsymbol{P}_{c}$ via (21) and utilize the rotational invariance in (25) to get the initial estimates of nominal DOAs $\left\{\widehat{\theta}_{k}\right\}_{k=1}^{K}$.

Step 2 Initialize the direction matrix $\widehat{B}_{1}$ and the matrix $\widehat{\boldsymbol{G}}$ with the initial estimations $\left\{\widehat{\theta}_{k}\right\}_{k=1}^{K}$.

Step 3 Achieve the three slices $\widetilde{\boldsymbol{R}}, \widetilde{\boldsymbol{Y}}, \widetilde{\boldsymbol{Z}}$ by rearranging the received signal data according to (13), (17), (18).
Step 4 Update $\boldsymbol{B}_{1}, \boldsymbol{G}$ and $\boldsymbol{V}$ until convergence via (28), (30), (32).

Step 5 According to (34), (39) and (40), the nominal DOAs can be achieved.

Remark 1 The number of CD sources is supposed to be known in this paper. If the exact number of CD sources is assumed to be unknown beforehand, we can determine the proper number of signals via the methods proposed in [32-34].

\section{Analysis of the proposed algorithm}

\subsection{Complexity analysis}

Firstly, we obtain the initial estimations of nominal DOAs via PM, the complexity is about $O\left(2 K^{2} Q+K Q(M P-\right.$ $\left.K)+3 K^{2}(M P-M)+3 K^{3}\right)$. The complexity of each iteration via TALS is $O\left(5 K^{2}(M P+M Q+P Q)+3 K^{3}+\right.$ $3 M P Q)$. And the proposed fast PARAFAC algorithm can reduce iteration times, which enables the proposed algorithm to have a lower complexity than that of conventional PARAFAC algorithms. It is shown obviously in Fig. 3 that PM has a lower complexity than each iteration of the PARAFAC algorithm.

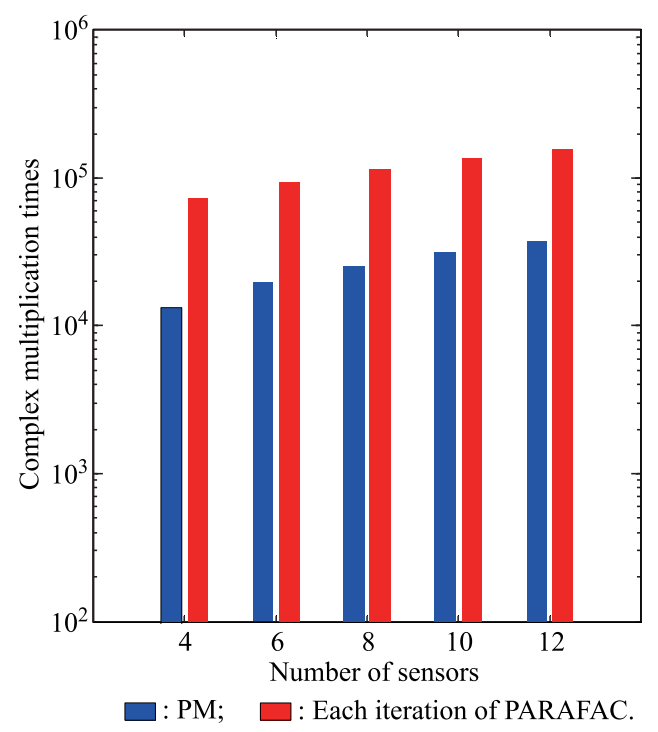

Fig. 3 Complexity of each iteration of PM and PARAFAC ( $K=$ $2, P=5, Q=300$ )

The comparison of complexity between the fast PARAFAC algorithm and the PARAFAC algorithm versus snapshots is illustrated in Fig. 4. The iteration times of the proposed algorithm and the conventional PARAFAC are set as 50 and 225 on average according to the convergence analysis in Section 5. 


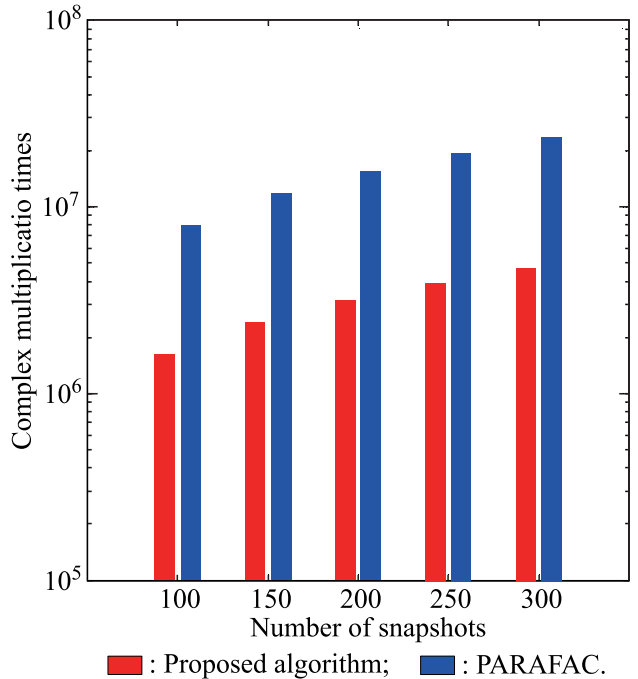

Fig. 4 Total complexity of the proposed algorithm and PARAFAC $(M=6, P=5, K=2)$

\subsection{Advantages of the proposed algorithm}

The main advantages of the proposed algorithm are summarized as follows:

(i) The proposed fast PARAFAC algorithm can achieve a fast convergence by utilizing PM to initialize the directional matrices, which can dispense with the high complexity caused by the random initialization. As compared with the conventional PARAFAC algorithm, the proposed algorithm can achieve the DOA estimates with no performance degradation, which is exhibited later in Section 5.

(ii) The proposed fast PARAFAC algorithm has superior estimation accuracy shown, as compared with ESPIRT-CD [13] and PM-CD [22] algorithm.

(iii) The proposed algorithm can be directly applied to the scenario with multiple sources, where sources have different angular distribution shapes, which can be proved later in Section 5.

\section{Simulation results}

In this section, we present the numerical simulation results to illustrate the performance of the proposed fast PARAFAC algorithm. The root mean squared error (RMSE) is adopted as the performance metric, which is given by

$$
\mathrm{RMSE}=\frac{1}{K} \sum_{k=1}^{K} \sqrt{\frac{1}{L} \sum_{l=1}^{L}\left(\widehat{\theta}_{k, l}-\theta_{k}\right)^{2}}
$$

where $L$ is the times of Monte-Carlo simulations, $\widehat{\theta}_{k, l}$ is the estimate of the $k$ th nominal DOA $\theta_{k}$ for the $l$ th MonteCarlo trial. The signal-to-noise ratio (SNR) is defined as
$\mathrm{SNR}=\rho_{k} / \sigma_{n}^{2}$, in which $\rho_{k}=\mathrm{E}\left\{|v(k)|^{2}\right\}$ is the signal power of the $k$ th source.

It is also assumed that there are two CD sources. One of the sources is assumed to be uniformly coherently distributed (UCD) with the deterministic angular distribution density function

$$
g_{1}\left(\theta ; \boldsymbol{\eta}_{1}\right)=\frac{1}{2 \sqrt{3} \sigma_{1}} \operatorname{Rect}\left[\theta_{1}-\sqrt{3} \sigma_{1}, \theta_{1}+\sqrt{3} \sigma_{1}\right]
$$

where $\theta_{1}$ and $\sigma_{1}$ are the nominal DOA and angular spread of the $\mathrm{CD}$ source, respectively. The rectangular window function $\operatorname{Rect}[c, d]$ is equal to one inside $[c, d]$ and is equal to zero elsewhere. The other one is assumed to be Gaussian coherently distributed (GCD) with the deterministic angular distribution density function

$$
g_{2}\left(\theta ; \boldsymbol{\eta}_{2}\right)=\frac{1}{\sqrt{2 \pi} \sigma_{2}} \exp \left\{-0.5\left(\theta-\theta_{2}\right)^{2} / \sigma_{2}^{2}\right\}
$$

where $\theta_{2}$ and $\sigma_{2}$ are the nominal DOA and angular spread of the CD source, respectively. The nominal DOAs $\theta_{1}$ and $\theta_{2}$ are taken as $30^{\circ}$ and $70^{\circ}$ while the angular spreads are set as $\sigma_{1}=1^{\circ}$ and $\sigma_{2}=1.5^{\circ}$.

Unless otherwise stated, in all the simulations, the multiple-invariance array is composed of $P=5$ ULAs, where each ULA consists of $M=6$ sensors with halfwavelength inter-element spacing. The displacement between each subarray is $d=\lambda / 20$. The number of snapshots is $Q=300$. The TALS algorithm for fitting the PARAFAC model is initialized with the PM method.

In the first example, we run 100 Monte-Carlo trials and illustrate the estimation results in Fig. 5. The SNR is set as $30 \mathrm{~dB}$. It is shown clearly that the fast PARAFAC algorithm can obtain valid nominal DOA estimates in the case of multiple CD sources which have different angular distribution shapes.



Fig. 5 Estimation results of 100 Monte-Carlo trials 
In the second example, we compare the iteration times of the fast PARAFAC algorithm and the PARAFAC algorithm in Fig. 6 where the SNR is set as $10 \mathrm{~dB}$. The sum of squared residuals (SSR) is defined as

$$
\mathrm{SSR}=\sum_{m=1}^{M} \sum_{q=1}^{Q} \sum_{p=1}^{P}\left[\widetilde{r}_{m, q, p}-\sum_{k=1}^{K}\left[\widehat{\boldsymbol{B}}_{1}\right]_{m, k}[\widehat{\boldsymbol{G}}]_{p, k}[\widehat{\boldsymbol{V}}]_{k, q}\right]^{2}
$$

where $\widetilde{r}_{m, q, p}$ denotes the received data.

Meanwhile, we define differential sum of squared residuals (DSSR) as

$$
\mathrm{DSSR}=\mathrm{SSR}_{p}-\mathrm{SSR}_{0}
$$

where $\mathrm{SSR}_{p}$ stands for the SSR of the $p$ th iteration and $\mathrm{SSR}_{0}$ stands for the SSR of the convergence condition. Due to the initialization via the PM method, the proposed algorithm has a fast convergence in Fig. 6. It is explicitly exhibited in Fig. 4 that the total iteration time of the fast PARAFAC algorithm is less than that of the conventional PARAFAC algorithm, which endows the proposed algorithm a much lower computation complexity.



Fig. 6 Analysis of iteration times via the proposed algorithm and PARAFAC

In the third example, provide the comparison of the estimation accuracy among the proposed algorithm, the PARAFAC algorithm, PM-CD [22] and ESPRIT-CD [13] algorithm by using 500 Monte-Carlo trials. Fig. 7 depicts the RMSE performance of the algorithms and it shows clearly that the proposed algorithm possesses the same estimation performance with the conventional PARAFAC algorithm and outperforms ESPRIT-CD [13] and PM-CD [22].

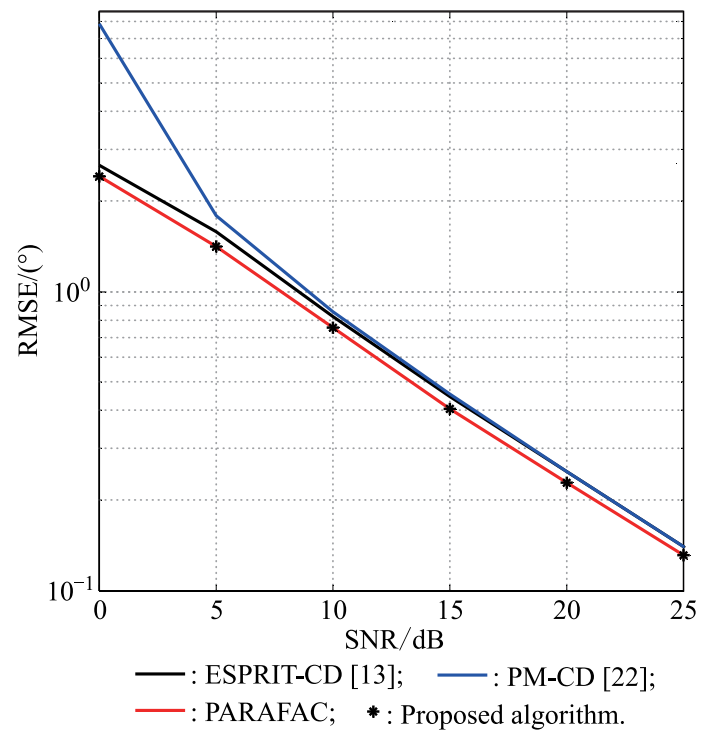

Fig. 7 Performance comparison versus SNR

In the fourth example, we study the DOA estimation performance of the fast PARAFAC algorithm with different numbers of sensors in Fig. 8 and Fig. 9. With $M$ or $P$ increasing, the proposed algorithm can obtain improved estimation performance because of diversity gain. In the last example, the RMSE performance of the proposed fast PARAFAC algorithm, the PARAFAC algorithm, ESPRITCD [13] and PM-CD [22] algorithm versus the number of snapshots is presented in Fig. 10, where SNR=20 dB and 1000 Monte-Carlo simulations are used. It is shown obviously that the proposed algorithm outperforms ESPRITCD [13] and PM-CD [22] in terms of estimation performance. In addition, Fig. 11 exhibits that the proposed algorithm can obtain better DOA estimates with $Q$ increasing.

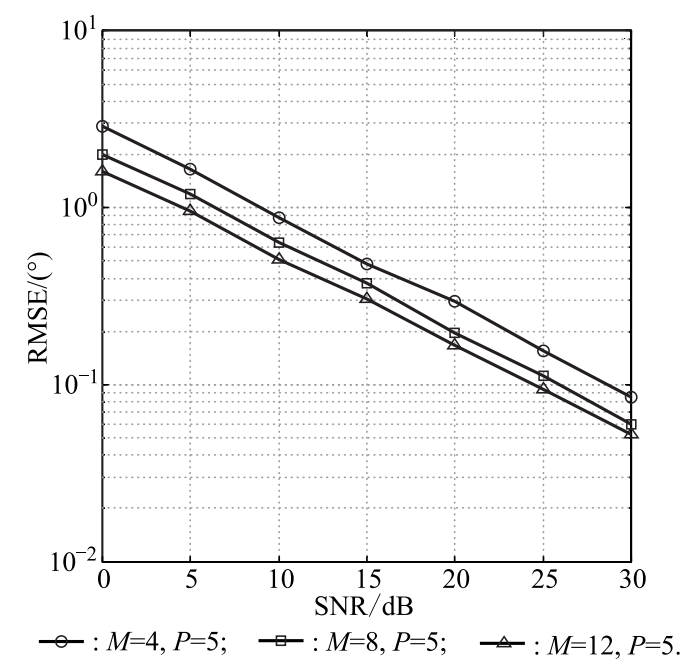

Fig. 8 Performance of the proposed algorithm versus $M$ 


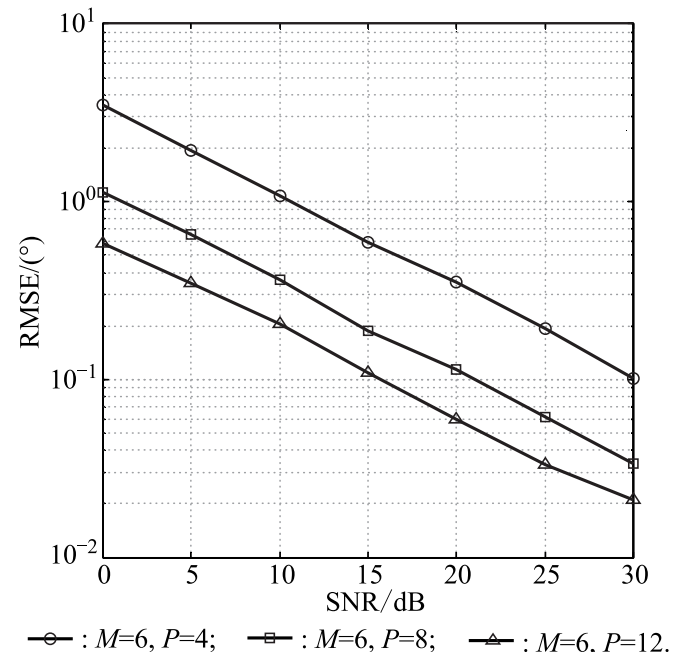

Fig. 9 Performance of the proposed algorithm versus $P$

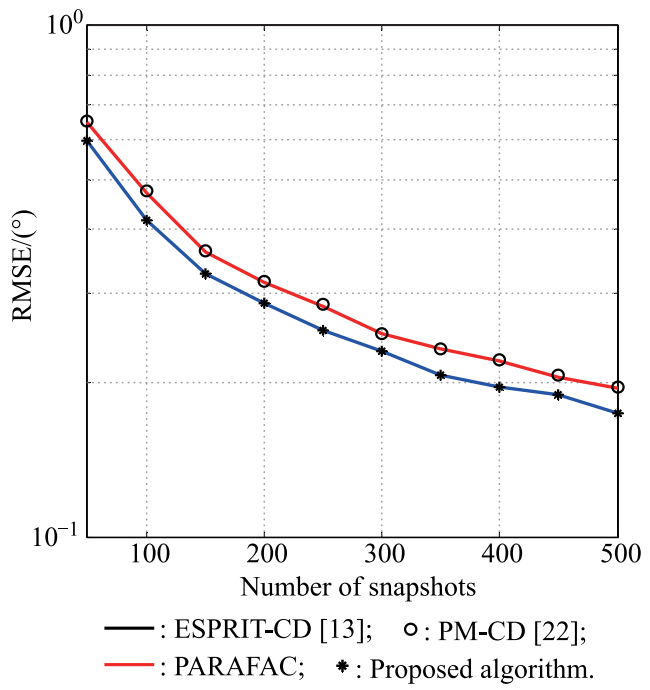

Fig. 10 Performance comparison versus the number of snapshots

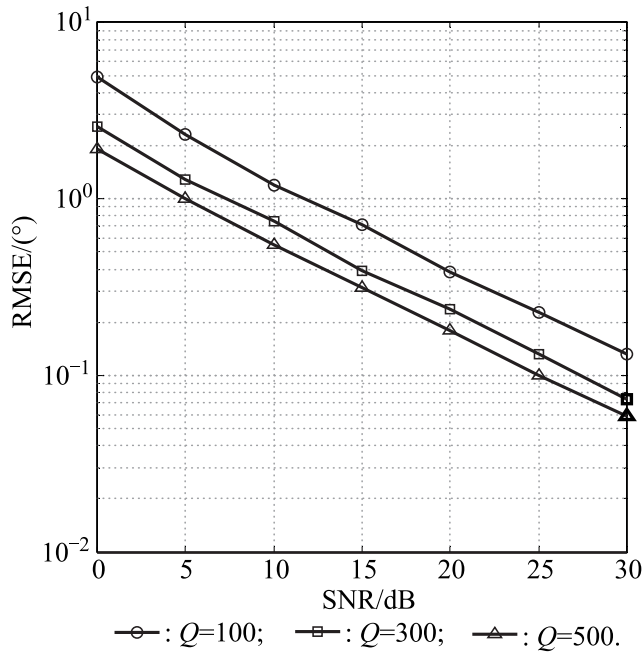

Fig. 11 Performance of the proposed algorithm versus $Q$

\section{Conclusions}

In this paper, we have shown that the nominal DOA estimation problem of $\mathrm{CD}$ sources can be accomplished by the fast PARAFAC decomposition technique. The proposed algorithm fully capitalizes on the approximate multipleinvariance property of the observed data with a special array geometry. Nominal DOA estimates are obtained by the aid of the uniqueness of the low-rank three-way array decomposition and the TALS regression. The high complexity can dramatically reduce by initializing the direction matrix with the PM method. In terms of nominal DOA estimation performance, simulation results illustrate that the proposed algorithm outperforms ESPRIT-CD [13] and PM$\mathrm{CD}$ [22] algorithm. Future work includes the research on the adaptive PARAFAC algorithm for CD sources tracking.

\section{References}

[1] VALAEE S, CHAMPAGNE B, KABAL P. Parametric localization of distributed sources. IEEE Trans. on Signal Processing, 1995, 43(9): $2144-12153$.

[2] ZOUBIR A, WANG Y. Efficient DSPE algorithm for estimating the angular parameters of coherently distributed sources. Signal Processing, 2008, 88(4): $1071-1078$.

[3] ZOUBIR A, WANG Y, CHARGÉ P. A modified COMETEXIP method for estimating a scattered source. Signal Processing, 2006, 86(4): $733-743$.

[4] BENGTSSON M, OTTERSTEN B. A generalization of weighted subspace fitting to full-rank models. IEEE Trans. on Signal Processing, 2001, 49(5): $1002-1012$.

[5] MENG Y, STOICA P. Estimation of direction of arrival of spatially dispersed signals in array processing. IEE Proceedings Radar Sonar and Navigation, 1996, 143(1): 1-9.

[6] CAFFERY J J, STUBER G L. Subscriber location in CDMA cellular networks. IEEE Trans. on Vehicular Technology, 1998, 47(2): 406-416.

[7] FISHLER E, HAIMOVICH A, BLUM R, et al. MIMO radar: an idea whose time has come. Proc. of the IEEE Radar Conference, 2004, 7(6): 71-78.

[8] FORTMANN T, BARSHALOM Y, SCHEFFE M. Sonar tracking of multiple targets using joint probabilistic data association. IEEE Journal of Oceanic Engineering, 1983, 8(3): $173-$ 184.

[9] TRUMP T, OTTERSTEN B. Estimation of nominal direction of arrival and angular spread using an array of sensors. Signal Processing, 1996, 50(1/2): 57-69.

[10] SCHMIDT R O. Multiple emitter location and signal parameter estimation. IEEE Trans. on Antennas \& Propagation, 1986, 34(3): $276-280$.

[11] ROY R, KAILATH T. ESPRIT-estimation of signal parameters via rotational invariance techniques. IEEE Trans. on Acoustics Speech \& Signal Processing, 1990, 37(7): 984-995.

[12] ASTELY D, OTTERSTEN B. The effects of local scattering on direction of arrival estimation with MUSIC. IEEE Trans. on Signal Processing, 1999, 47(12): 3220-3234.

[13] SHAHBAZPANAHI S, VALAEE S, BASTANI M H. Distributed source localization using ESPRIT algorithm. IEEE Trans. on Signal Processing, 2001, 49(10): 2169-2178.

[14] ZOUBIR A, WANG Y, CHARGE P. Efficient subspace-based estimator for localization of multiple incoherently distributed sources. IEEE Trans. on Signal Processing, 2008, 56(2): $532-$ 
542.

[15] BOUJEMÂA H. Extension of COMET algorithm to multiple diffuse source localization in azimuth and elevation. European Trans. on Telecommunications, 2005, 16(6): 557-566.

[16] HAN Y, WANG J, ZHAO Q. Decoupled parameter estimation for coherently distributed source. Berlin: Springer, 2014.

[17] FERREOL A, BOYER E, YIN X, et al. A simple decoupled estimation of DOA and angular spread for single spatially dispersed sources. Proc. of the 14th European Signal Processing Conference, 2006: 1-5.

[18] CAO R, GAO F, ZHANG X. An angular parameter estimation method for incoherently distributed sources via generalized shift invariance. IEEE Trans. on Signal Processing, 2016, 64(17): $4493-4503$.

[19] ZHANG G, TANG B. Decoupled estimation of 2D DOA for coherently distributed sources using 3D matrix pencil method. EURASIP Journal on Advances in Signal Processing, 2008, 2008(1): $1-7$.

[20] LEE J, SONG I, KWON H, et al. Low-complexity estimation of 2D DOA for coherently distributed sources. Signal Processing, 2003, 83(8): $1789-1802$.

[21] HU A, LV T, GAO H, et al. An ESPRIT-based approach for 2D localization of incoherently distributed sources in massive MIMO systems. IEEE Journal of Selected Topics in Signal Processing, 2014, 8(5): 996-1011.

[22] ZHENG Z, LI G, TENG Y. 2D DOA estimator for multiple coherently distributed sources using modified propagator. Circuits, Systems, and Signal Processing, 2012, 31(1): 255-270.

[23] BENGTSSON M, OTTERSTEN B. Rooting techniques for estimation of angular spread with an antenna array. Proc. of the 47th IEEE Vehicular Technology Conference, 1997: $1158-$ 1162.

[24] ZOUBIR A, WANG Y. Robust generalised Capon algorithm for estimating the angular parameters of multiple incoherently distributed sources. IET Signal Processing, 2008, 2(2): $163-$ 168.

[25] SIESKUL B T. An asymptotic maximum likelihood for joint estimation of nominal angles and angular spreads of multiple spatially distributed sources. IEEE Trans. on Vehicular Technology, 2010, 59(3): $1534-1538$.

[26] SIDIROPOULOS N D, BRO R, GIANNAKIS G B. Parallel factor analysis in sensor array processing. IEEE Trans. on Signal Processing, 2000, 48(8): 2377 -2388.

[27] SIDIROPOULOS N D, GIANNAKIS G B, BRO R. Blind parafac receivers for DS-CDMA systems. IEEE Trans. on Signal Processing, 2000, 48(3): 810-823.

[28] VDV A J. Algebraic methods for deterministic blind beamforming. Proceedings of the IEEE, 1998, 86(10): 1987-2008.

[29] LI J, ZHANG X, CHEN H. Improved two-dimensional DOA estimation algorithm for two-parallel uniform linear arrays using propagator method. Signal Processing, 2012, 92(12): $3032-3038$.

[30] MARCOS S, MARSAL A, BENIDIR M. The propagator method for source bearing estimation. Signal Processing, 1995, 42(2): $121-138$.

[31] KRUSKAL J B. Three-way arrays: rank and uniqueness of trilinear decompositions, with application to arithmetic complexity and statistics. Linear Algebra \& Its Applications, 1977, 18(2): $95-138$.

[32] BRO R, KIERS H A L. A new efficient method for determining the number of components in PARAFAC models. Journal of Chemometrics, 2003, 17(5): 274-286.

[33] LIU K, SO H C, COSTA J P C L D, et al. Core consistency diagnostic aided by reconstruction error for accurate enumeration of the number of components in parafac models. Proc. of IEEE International Conference on Acoustics, Speech and Signal Processing, 2013: 6635-6639.

[34] XIN J, ZHENG N, SANO A. Simple and Efficient nonparametric method for estimating the number of signals without eigen decomposition. IEEE Trans. on Signal Processing, 2007, 55(4): $1405-1420$.

\section{Biographies}

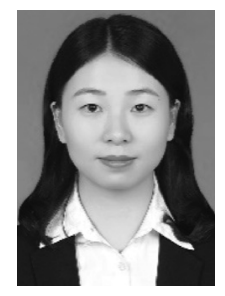

CHENG Qianlin was born in 1993. She received her B.S. degree from the College of Electronic and Information Engineering of Nanjing University of Aeronautics and Astronautics, Nanjing, China in 2015, where she is currently pursing her Ph.D. degree. Her research interests include array signal processing, distributed source localization, etc.

E-mail: chengqianlin_nuaa@163.com

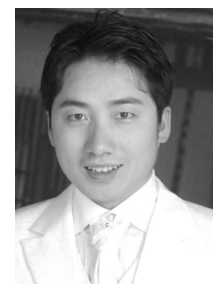

ZHANG Xiaofei was born in 1977. He received his Ph.D. degree in communication and information systems from Nanjing University of Aeronautics and Astronautics in 2005. Now, he is a professor in the Electronic Engineering Department, Nanjing University of Aeronautics and Astronautics, Nanjing, China. His research focuses on array signal processing and communication signal processing. E-mail: fei_zxf@163.com

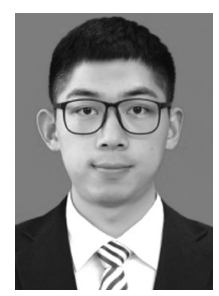

CAO Renzheng was born in 1991. He received his B.S. degree from the College of Electronic and Information Engineering of Nanjing University of Aeronautics and Astronautics, Nanjing, China in 2012. He is currently pursing his Ph.D. degree in the College of Electronic and Information Engineering of Nanjing University of Aeronautics and Astronautics, Nanjing, China. His research interests include array signal processing, radar signal processing, wireless communication, etc.

E-mail: caorenzheng@nuaa.edu.cn 